\title{
IMPLICAÇÕES PARA O PROCESSO DE ENFERMAGEM NA UNIDADE DE TERAPIA INTENSIVA
}

\author{
Lorraine Barreto Morais
}

Acadêmica em Enfermagem/Institutos Superiores de Ensino do CENSA/ISECENSA/RJ

lorraine_morais@yahoo.com.br

Marina Santos Cezário

Acadêmica em Enfermagem/Institutos Superiores de Ensino do CENSA/ISECENSA/RJ

marinascezario@gmail.com

\section{Aline Siqueira de Azevedo}

Especialista em Enfermagem Intensivista/Universidade Estadual do Rio de Janeiro/UERJ/RJ

enfsiqueira@gmail.com

\section{Letycia Sardinha Peixoto Manhães}

Mestre em Ciências do Cuidado em Saúde - Universidade Federal Fluminense - UFF/RJ

letyciasardinha@gmail.com

\section{RESUMO}

A ciência da enfermagem é conceituada como uma ampla formação teórica, e o Processo de Enfermagem (PE) é um instrumento que permite que essa estrutura seja aplicada à prática da enfermagem - ou seja, é a estratégia de solução dos problemas do paciente. Tal metodologia torna-se essencial para o processo de trabalho do enfermeiro, principalmente em Unidades de Terapia Intensiva (UTI), colaborando para a segurança do cuidado prestado aos pacientes que se encontram em estado crítico de saúde, pois quanto maior o número de necessidades afetadas do cliente, maior a necessidade de se planejar a assistência. Assim sendo, essa pesquisa teve como objetivo descrever a atuação do enfermeiro na Unidade de Terapia Intensiva frente à Sistematização da Assistência de Enfermagem em um hospital de referência e grande porte, situado no município de Campos dos Goytacazes/RJ. Foi adotada como metodologia a pesquisa descritiva com abordagem qualitativa. Numa primeira análise, constatou-se que a implementação da SAE ocorre de forma ainda bastante fragmentada, o que indica a necessidade de reorganização dessa metodologia de assistência. Diante disso, elaboramos um instrumento de evolução do enfermeiro com objetivo de facilitar sua rotina e incentivar o desenvolvimento da SAE, tendo como base as cinco fases do Processo de Enfermagem.

Descritores: Assistência de Enfermagem, Processos de Enfermagem, Unidades de Terapia Intensiva.

\begin{abstract}
The nursing science is conceptualized as a comprehensive theoretical training and the Nursing Process (NP) is an instrument that allows this structure to be applied to the nursing practice - that is, the solution strategy of the patient's problems. This methodology becomes essential for the working process of nurses, especially in Intensive Care Units (ICU), contributing to the safety of care provided to patients who are in critical health condition, because the higher the number of affected customer needs, the greater the need to plan assistance. Therefore, this study aimed to describe a nurse's performance on the Intensive Care Unit front of the systematization of nursing care in hospital Reference sized and large hum, located in the municipality of Campos dos Goytacazes / RJ. The descriptive research was adopted as methodology with a qualitative approach. At first sight, it was found that the implementation of the NCS is still a quite fragmented form, which indicates the need for reorganization of this methodology assistance. Therefore, we developed an
\end{abstract}


evolving instrument nurse in order to facilitate your routine and encourage the development of SAE, based on the five stages of Nursing Process .

Descriptors: Nursing Care, Nursing Process, Intensive Care Units.

\section{INTRODUÇÃO}

A Enfermagem Moderna, que tem como precursora Florence Nightingale, deu origem a sua caminhada para adoção de uma prática sustentada em conhecimentos científicos, deixando progressivamente a postura de atividade caritativa, intuitiva e empírica. Desta forma, a resolução julgada como ideal a partir da década de 1950, para o cuidado aos clientes em estado grave, passou a ser o plano de cuidados. No entanto, o mesmo já era indispensável, segundo os ensinamentos de Florence (DANIEL, 1979; HORTA,1979).

Conforme citado em Taveira et al. (2012), Nightingale estabeleceu um dos princípios básicos da terapia intensiva moderna, afastando os pacientes mais graves e colocando-os em uma situação que favorecia o cuidado por meio da observação constante, nascendo assim às primeiras estações de Unidade de Terapia Intensiva (UTI).

Mais a frente, na década de 1970 no Brasil, com o objetivo de atender pacientes em estado agudo ou crítico sujeitos à instabilidade de funções vitais, mas com possibilidades de recuperação, as Unidades de Terapia Intensiva foram criadas. Portanto, esses pacientes demandam assistência médica e de enfermagem constante e especializada, necessitando também do apoio de equipamentos especiais de diagnóstico e tratamento (TRANQUITELI \& CIAMPONE, 2007).

Pedreira (2009), conceitua o cuidado de enfermagem ofertado a pacientes em terapia intensiva como sendo um processo que envolve tomada de decisões, que implicam em questões éticas, opções terapêuticas e demandas gerenciais. $\mathrm{Na}$ análise clínica, as evidências indicam se as condutas são eficazes e auxiliam na dispensa das melhores práticas, sendo elas: o cuidado certo, no momento certo, da maneira certa, para o paciente certo, objetivando alcançar os melhores resultados possíveis. "A partir disso, o julgamento clínico auxilia na análise situacional para definição das ações a serem adotadas para determinados pacientes" (COLAÇO, et. al, 2015).

Dos Santos (2014), contextualiza a enfermagem como uma profissão dinâmica, necessitando de uma estrutura que seja eficaz, refletindo tal dinamismo. O Processo de Enfermagem (PE) é considerado a metodologia de trabalho mais conhecida e aceita no mundo, facilitando a troca de informações entre enfermeiros de várias instituições. Tal metodologia apresenta-se como instrumento organizacional e estratégia de implementação do cuidado, visto que a enfermagem tem como foco de estudos e práticas o cuidado integral e individual dos clientes, que utiliza a tecnologia somada às relações interpessoais para organizar e planejar as demandas de cuidado, gerenciais e científicas (AMANTE, ROSSETTO \& SCHNEIDER, 2009).

De Oliveira \& Evangelista (2010) apontam que as teorias de enfermagem foram desenvolvidas com o objetivo de organizar e sistematizar todas as questões onde a atividade profissional esteja presente, gerando conhecimentos que sustentarão e subsidiarão a prática do enfermeiro. É através da aplicação dessa teoria à prática é que se dá o $\mathrm{PE}$, e para que o mesmo seja aderido pela equipe de enfermagem e verdadeiramente otimize e qualifique o cuidado prestado ao cliente é necessário que se adquira, concomitantemente, uma assistência de enfermagem sistematizada. Logo, a Sistematização da Assistência de Enfermagem (SAE) e o $\mathrm{PE}$ devem ser implementados conjuntamente para que os resultados sejam positivos e benéficos tanto para o cliente quanto para o profissional de enfermagem.

Truppel et. al (2009) enfatizam que SAE se mostra como uma metodologia para planejar e sistematizar a assistência, baseada nos princípios do método científico. Seus objetivos principais são constatar as situações de saúde-doença e as necessidades de cuidado de enfermagem, e também de assistir as 
intervenções de promoção, prevenção, recuperação e reabilitação do estado de saúde do indivíduo, família e comunidade.

A implantação da SAE segundo Remizoski, Rocha \& Vall (2010), exige uma metodologia para ser efetivada. Um método que pode ser adotado para firmar essa prática é o Processo de Enfermagem, que é formado por etapas inter-relacionadas, tais como: Histórico, onde se faz a coleta de dados do paciente; Diagnósticos de Enfermagem; Prescrição, que vem a ser o planejamento para se obter o cuidado adequado; Implementação, que são as ações de enfermagem baseadas no programação já pré-estabelecida e a Avaliação dos resultados obtidos

A primeira fase do PE é a investigação ou histórico de enfermagem, que de acordo com Tannure \& Gonçalves (2011), serão avaliadas as condições de saúde do cliente e identificado os problemas, percepções e expectativas que demandam ações de enfermagem, além de organizar, analisar e sintetizar os dados coletados que servem de ponto inicial no diagnóstico de uma doença.

Após a coleta de dados, é realizado o exame físico, como apresentado por Luiz et. al (2010) que através das técnicas de inspeção, ausculta, palpação e percussão, será realizado o levantamento dos dados sobre o estado de saúde do paciente e anotações do estado clínico, que servirão como subsídio para a confirmação de um diagnóstico adequado.

De acordo com Barros \& Lopes (2010), o Diagnóstico de Enfermagem, segunda etapa do PE, é realizada a interpretação dos dados coletados, onde os mesmos serão analisados detalhadamente com o objetivo de elaborar um diagnóstico adequado. Os autores ainda mencionam que a terceira etapa, planejamento de enfermagem, caberá ao enfermeiro estabelecer quais as condutas serão implementadas, e avaliar os resultados, cabendo ao profissional à liderança na execução dessa e das demais etapas, com o intuito de alcançar os resultados esperados. A implementação, quarta etapa, significa a introdução dos planejamentos e/ou protocolos. Tannure\& Gonçalves (2011) ainda relatam que a quarta fase, são ações prescritas e registradas pelo enfermeiro, que visam diminuir riscos, solucionar os diagnósticos de enfermagem e promover a saúde.

De acordo com Luiz et. al (2010), para que a implementação ocorra, é fundamental o provimento recursos suficientes para atender as necessidades específicas da cada cliente, de forma individualizada, humanizada e independente de prescrição médica. A avaliação é a quinta etapa do PE, é a evolução clínica do cliente e o enfermeiro pode fundamentar medidas corretivas ou reavaliar planos de cuidados, caso seja necessário.

A evolução de enfermagem de acordo com Cianciarullo et. al (2008), é o registro elaborado posteriormente a avaliação do estado clínico geral do paciente, que objetiva nortear o planejamento da assistência a ser prestada e relatar resultados e condutas de enfermagem implementadas.

Garcia \& Nóbrega (2009), destacam que a aplicação da SAE envolve mais que um conjunto de passos a serem seguidos, cobrando do profissional maior familiaridade dos diagnósticos de enfermagem e sensibilidade para adequar as necessidades do cliente às condições de trabalho, tornando-as menos simples do que sugere a teoria.

Reforçando a necessidade do planejamento da assistência de enfermagem, a Resolução do Conselho Federal de Enfermagem (COFEN) no 358/209 reafirma que a Sistematização da Assistência de Enfermagem é um instrumento privativo do processo de trabalho do enfermeiro, e conforme o artigo $1^{\mathrm{o}}$, a sua implementação deve ocorrer em toda instituição de saúde, pública e privada.

As Unidades de Terapia Intensiva são conceituadas pela RDC n ${ }^{\circ}$ 07/2010 como setores hospitalares que atendem pacientes graves, instáveis, ou de alto risco. Desta forma, esse ambiante carece de assistência à saúde de forma ininterrupta, agregando equipamentos tecnológicos específicos e recursos humanos especializados. 
Amante, Rossetto \& Schneider (2013) indicam que a necessidade de ação rápida, segura e efetiva da equipe de enfermagem e o longo tempo de permanência desses pacientes no ambiente hospitalar na UTI, fazem com que o Processo de Enfermagem se torne fundamental devido o agravamento da situação de saúde dos pacientes internados, dificultando a entrevista e exigindo uma observação e exames físicos adequados.

Reafirmando a importância da SAE, Massaroli, A., Massaroli, R., \& Martini (2013) defendem como prática fundamental para o processo de trabalho do enfermeiro o aprimoramento do PE na UTI, colaborando com a garantia do cuidado dispensado aos pacientes que se encontram em estado crítico de saúde.

Rocha et al. (2012), afirmam que a identificação das necessidades da assistência de enfermagem como uma das etapas do processo assistencial do enfermeiro é indispensável, em especial para pacientes em estado grave com risco de morte, no sentido de auxiliar a prescrição de enfermagem e a avaliação do cuidado.

Nesse contexto Malucelli et. al (2010), reforçam é preciso levar em consideração que o cuidado do profissional de enfermagem é decorrente de um instrumental tecnológico desenvolvido ao longo da formação profissional e aperfeiçoado em atividades de educação permanente, que resultem numa prática reflexiva e crítica, não sendo um fenômeno natural e, sim, resultante de um empreendimento humano.

Brasil (2009) propõe que os processos de educação dos trabalhadores da saúde se façam a partir da problematização do processo de trabalho. Os processos de educação permanente em saúde objetivam a transformação das práticas profissionais e da própria organização do trabalho. A educação permanente é feita a partir dos problemas enfrentados na realidade e leva em conta os conhecimentos e as experiências que as pessoas já têm, é baseada na aprendizagem significativa e na possibilidade de transformar as práticas profissionais e pode ser entendida como aprendizagem-trabalho, ou seja, ela acontece no cotidiano das pessoas e das organizações.

Diante disso, há uma problemática acerca da implementação do PE, podendo ser demostrada através de estudo realizado por Takahashi (2008), onde ele menciona que a formação possivelmente deficitária gera uma não responsabilização do modelo assistencial para a SAE, e o desconhecimento sobre o assunto se torna um fator problema onde um dos motivos fundamentais para a falta de comprometimento na execução do PE em algumas instituições de saúde, venha ser resultante da falta de conhecimento à respeito do mesmo, tal fato resulta além de execução descompromissada, também na não implementação desse modelo assistencial.

De acordo Andrade \& Vieira (2005, p. 262) "a formação acadêmica dos enfermeiros, muitas vezes contribui para que estes não busquem nem apliquem uma assistência sistematizada". Isso acontece porque durante as aulas práticas observa-se uma maior preocupação em desenvolver habilidades técnicas, diminuindo-se o interesse pelo planejamento dos cuidados.

A sobrecarga de trabalho nas instituições de saúde devido à deficiência de recursos humanos se mostra um agravante frente ao aumento na demanda de atendimento, onde a prestação de serviço em meio a essa problemática pode tornar a assistência defasada. Nesse contexto de precarização, é apontada a escassez de recursos humanos nos serviços de enfermagem, que operam geralmente com um contingente de profissionais aquém das necessidades, visando atender a uma demanda crescente de pacientes (PIMPÃO et al., 2010).

Brasil (2009) descreve que a educação permanente em saúde possibilita transformar as práticas profissionais e a própria organização do trabalho. Sendo assim, podemos entender que teoria e prática caminham juntas. Nesse sentido, Ruthes \& Cunha (2008) afirmam que a educação continuada potencializa a articulação de saberes voltados aos novos papéis e circunstâncias, como estratégia de valorização das competências pessoais, demonstrando que o processo de educação continuada na enfermagem tem como função formar profissionais com um olhar crítico e ampliado de suas ações, construindo assim, seu exercício diário baseado na articulação teórico-prática.

Persp. online: biol. \& saúde, Campos dos Goytacazes, 19 (5), 35-52, 2015

seer.perspectivasonline.com.br 
A implementação do Processo de Enfermagem tem sofrido grande resistência, predominando na assistência direta um cuidado de enfermagem pautado nas atividades mais técnicas, tendo como consequência uma prática mecanicista e administrativa, onde o foco do serviço é o cumprimento dessas atividades, causando prejuízo na assistência direcionada às necessidades do paciente. Sendo assim, osprofissionais enfermeiros, envolvidos na mecânica dos afazeres rotineiros e pela cobrança de resultados positivos, parecem não incorporar o PE como instrumento de trabalho, que deve adequar-se às necessidades da clientela assistida e aos objetivos que vislumbrem o cuidado humano digno (ALVES, LOPES \& JORGE, 2008).

Em síntese, para Pimpão et al. (2010), a conscientização do profissional enfermeiro acerca do seu papel como líder de equipe, torna-se imprescindível uma vez que possibilita a promoção de mudanças no cenário da prática assistencial e transforma o atual sistema de assistência à saúde.

De acordo com o contexto exposto, temos como questões norteadoras: O enfermeiro utiliza a Sistematização da Assistência de Enfermagem nos cuidados com o paciente da Unidade de Terapia Intensiva? Como o enfermeiro compreende o Processo de Enfermagem na sua assistência diária ao paciente da Unidade de Terapia Intensiva? Como o enfermeiro articula o Processo de Enfermagem com sua assistência diária ao paciente na Unidade de Terapia Intensiva?

A realização dessa pesquisa teve como objetivo descrever a atuação do enfermeiro da UTI frente a SAE, identificando seu conhecimento e compreendendo de que forma é articulada no cotidiano da assistência em um hospital de grande porte e referência da região, em Campos dos Goytacazes, Rio de Janeiro. Além disso, foi realizada a elaboração de um instrumento de evolução do enfermeiro para o incentivo do desenvolvimento da Sistematização da Assistência de Enfermagem com base nas cinco fases do Processo de Enfermagem.

\section{METODOLOGIA}

\subsection{Tipo de Estudo}

Trata-se de um estudo descritivo com caráter qualitativo, que segundo Gil (2008), descreve as características de determinadas populações ou fenômenos, sendo que uma de suas peculiaridades está na utilização de técnicas padronizadas de coleta de dados, tais como o questionário e a observação sistemática.

E de acordo com Polit, Becker \& Hungler (2011) a pesquisa qualitativa tende a salientar os aspectos dinâmicos, holísticos e individuais da experiência humana, para apreender a totalidade no contexto daqueles que estão vivenciando o fenômeno. Foi realizada uma pesquisa de campo que, como citado por Lakatos \& Marconi (2011) é uma forma de levantamento de dados no próprio local onde ocorrem os fenômenos, através da observação direta, entrevistas e medidas de opinião.

\subsection{Local de Estudo}

O estudo foi desenvolvido na Unidade de Terapia Intensiva de um hospital público referência no atendimento de emergência em Campos dos Goytacazes e nas regiões Norte e Noroeste Fluminense. O hospital, que existe há 59 anos, é reconhecido pelo Ministério da Saúde com a classificação de Nível III (máximo) em atendimento de emergência.

O hospital funciona com um quadro de cerca de 1.500 funcionários, composto por um prédio principal, com cinco andares, e dois anexos. Sua Unidade de Terapia Intensiva possui 24 leitos e conta com equipe: médica, enfermagem e fisioterapia em regime de plantão de $24 \mathrm{~h}$ e serviços de nutrição, fonoaudiologia, serviço social e psicologia integrados. Optou-se pela determinada instituição devido o 
elevado quantitativo de leitos e consequente demanda de enfermeiros intensivistas e também por ser referência no atendimento de emergência na rede Sistema Único de Saúde (SUS).

\subsection{Amostra}

A UTI da instituição escolhida conta atualmente com um número de enfermeiros intensivistas igual a 21 , onde todos foram convidados a participar do presente estudo, que pretendeu alcançar um percentual de $100 \%$ a fim de garantir a fidedignidade da pesquisa.

Atendendo aos seguintes critérios de inclusão: trabalhar na Unidade de Terapia Intensiva e manifestar interesse em responder o questionário, e como critérios de exclusão, aqueles profissionais enfermeiros que estiverem de férias ou licença e os que no momento da coleta não estiverem presentes.

\subsection{Coleta de dados}

Foram utilizados os seguintes instrumentos:

- Entrevista (Apêndice A): a primeira parte continha perguntas fechadas para caracterização dos sujeitos sobre gênero, idade, tempo de experiência, especialização, entre outros. A segunda parte da entrevista contou com questões abertas acerca dos seguintes temas: De que forma enfermeiro utiliza a Sistematização da Assistência de Enfermagem nos cuidados com o paciente da Unidade de Terapia Intensiva? Como o enfermeiro compreende o Processo de Enfermagem na sua assistência diária ao paciente da Unidade de Terapia Intensiva? O enfermeiro articula o Processo de Enfermagem com sua assistência diária ao paciente na Unidade de Terapia Intensiva?

- Análise documental: compreendeu o acompanhamento através da leitura da evolução do enfermeiro, sete dias anteriores ao dia da coleta, a partir de um Roteiro de Análise da Evolução do Enfermeiro (Apêndice B), que auxiliou na análise e proporcionou a composição de uma evolução de enfermagem segundo a SAE. O processo de análise documental é citado por Oliveira (2007), como um processo de tratamento do material para armazenar as informações de maneira mais acessível, condensada e contextualizada socialmente.

Ao final da coleta de dados, foi construído um modelo de evolução do enfermeiro que visa o desenvolvimento da SAE baseado nas cinco fases do PE (Apêndice C), elaborado através do plano compartilhado de conhecimento que, segundo Freire (2011), consiste numa reflexão crítica acerca do ato para a construção de um novo saber, onde através de um compartilhamento de saberes e práticas haverá a revelação de uma ação já praticada por um ou ambos do processo. Esse instrumento será uma proposta de recomendação do estudo, sem nenhum compromisso da instituição com a aceitação e utilização do mesmo.

\subsection{Análise dos Dados}

A interpretação dos dados presentes na entrevista se fundamentou na Análise de Conteúdo proposta por Bardin (2011), que define a mesma como um conjunto de técnicas de análise das comunicações, visando obter por procedimentos sistemáticos e objetivos de descrição do conteúdo das mensagens, indicadores que permitam a inferência de conhecimentos relativos às condições de produção e recepção destas mensagens.

Segundo Cedón \& Meireles (2010), para utilizar o método é necessário criar categorias relacionadas ao objeto de pesquisa. As deduções lógicas ou inferências que serão obtidas a partir das categorias serão responsáveis pela identificação das questões norteadoras contidas no conteúdo das mensagens. 
Os dados foram analisados a partir da leitura dos resultados obtidos com a pesquisa seguindo os seguintes passos: codificação dos dados com identificação dos trechos que abordavam a SAE, sua compreensão e articulação; formação das categorias; e elaboração da identificação dos conjuntos de expressões que representaram o conhecimento e importância da utilização da SAE.

\subsection{Aspectos Éticos}

O presente estudo foi autorizado pelainstituição hospitalar que serviu como campo eaprovado pelo Comitê de Ética e Pesquisa do ISECENSA em 28 de novembro de 2014 sob o número de CAAE: 38866514.0 .0000 .5524 .

Foi solicitado aos participantes a leitura e posterior assinatura de um Termo de Consentimento Livre e Esclarecido antes de se iniciar qualquer fase da coleta de dados.

\section{RESULTADOS E DISCUSSÃO}

\subsection{Caracterização da amostra}

A Unidade de Terapia Intensiva, onde o estudo foi realizado, é composta por 21 (vinte e um) enfermeiros e integraram o estudo 17 (81\%) dos enfermeiros lotados no setor. Foi excluído 01 (um) profissional que se encontrava de licença especial, e 03 (três) se recusaram a participar da pesquisa. Do total de entrevistados, 02 (dois) são enfermeiros diaristas, e os demais plantonistas.

Em relação às características sociodemográficas da amostra, foi observada uma faixa etária entre 25 (vinte e cinco) a 56 (cinquenta e seis) anos. Quanto ao gênero, 09 (nove) eram do sexo feminino e 08 (oito) do sexo masculino. Relacionado ao tempo de graduação, evidenciou-se uma heterogeneidade, variando entre 02 (dois) e 29 (vinte e nove) anos. Pôde ser notado também que o tempo médio dos enfermeiros na instituição corresponde a uma média de 09 (nove)anos, e no setor de terapia intensiva de 8,5 anos. A carga horária dos enfermeiros entrevistados equivale a 24 horas semanais, exceto os diaristas, que trabalham 06 horas diárias. Em relação ao nível de formação da amostra (figura 1), conclui-se que $71 \%$ tem alguma especialização na área de terapia intensiva, $18 \%$ referiram especializações em áreas distintas, $06 \%$ não possuem especialização e os outros $06 \%$ não responderam.

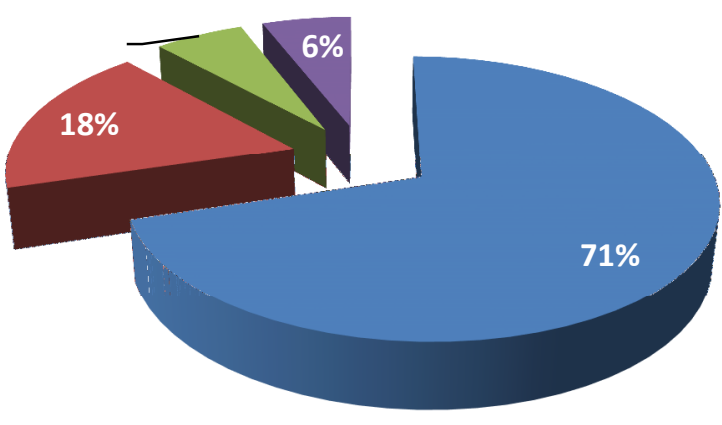

- Intensivistas

- Outras Especializações

- Sem Especialização

não responderam

Figura 1 - Especialização dos enfermeiros da Unidade de Terapia Intensiva em Hospital de referência e grande porte, situado no município de Campos dos Goytacazes, RJ - 2015 Fonte: Autoria Própria

Atualmente, o hospital estudado demonstra conformidade com relação ao dimensionamento de profissional enfermeiro, possuindo 24(vinte e quatro) leitos distribuídos entre 03 (três) ilhas, onde cada enfermeiro plantonista é responsável por 08 (oito) leitos, de acordo com o que prevê a Resolução da Diretoria Colegiada (RDC) n²6/2012 da Agência Nacional de Vigilância Sanitária (Anvisa), que determina

Persp. online: biol. \& saúde, Campos dos Goytacazes, 19 (5), 35-52, 2015

seer.perspectivasonline.com.br 
que a cada 10 (dez) leitos ou fração deve haver no mínimo 01 (um) Enfermeiro Assistencial, e ainda estabelece padrões mínimos para o funcionamento das UTI, e busca a redução de riscos aos pacientes, visitantes, profissionais e meio ambiente.

\subsection{Análise Documental}

Foi realizada a leitura do material disponível na instituição, tais como: Evolução de Enfermagem, Evolução diária do Enfermeiro Rotina, Prescrição de Enfermagem, Ficha de Admissão de Enfermagem e Prescrição Eletrônica de Enfermagem. Através destes, foi observado que as fases são realizadas, porém de forma indevida, onde pode-se mencionar a fragmentação entre as etapas através de impressos incompletos e/ou inadequados e pouco comprometimento por parte dos profissionais durante o preenchimento das evoluções de enfermagem, pois ainda que verbalizem sua importância, preenchem de forma deficiente os instrumentos disponíveis no setor.

Segundo a Resolução COFEN - 358/2009 que revoga a Resolução COFEN nº 272/2002, determina em seu artigo $1^{\circ}$ que é privativo ao enfermeiro a "implantação, planejamento, organização, execução e avaliação do processo de enfermagem, que compreende as seguintes etapas: histórico de enfermagem, exame físico, diagnóstico de enfermagem, prescrição de enfermagem e evolução".

Perante o exposto, a não realização de forma adequada do registro de enfermagem, conforme determina a resolução mencionada acima, transforma a SAE inacabada e inoperante, revelando uma contraposição entre o que é determinado e o que é aplicado. É importante acrescentar que no decorrer da realização da SAE muitos enfermeiros se esbarram com vários elementos cruciais na sua implantação, sendo necessária uma adaptação ao contexto do momento e do estabelecimento de saúde.

A partir da interpretação e análise dos dados dos enfermeiros entrevistados, emergiram três categorias: O Conhecimento do Enfermeiro da Unidade de Terapia Intensiva acerca da Sistematização da Assistência de Enfermagem, O Enfermeiro Frente à Sistematização da Assistência de Enfermagem X Processo de Trabalho da Enfermagem e Instrumentos para a Implementação da SAE na UTI: Um Produto de Pesquisa. $\mathrm{O}$ anonimato foi mantido e cada sujeito da amostra foi classificado com um codinome, como por exemplo, (enfermeiro A, B, C...).

\subsection{Categoria 1 - O Conhecimento do Enfermeiro da Unidade de Terapia Intensiva Acerca da Sistematização da Assistência de Enfermagem}

A SAE, enquanto processo organizacional é capaz de conceder subsídios para a evolução de métodos interdisciplinares e humanizados de cuidado com os indivíduos. Ela faz parte de um processo que vem sendo desenvolvido ao longo do tempo por enfermeiros comprometidos em melhorar cada vez mais o cuidado prestado ao paciente, pois vislumbram a importância de um cuidado interativo, complementar e multiprofissional (DOS SANTOS, 2014).

Os enfermeiros entrevistados expressaram ter entendimento do conceito de SAE, podendo ser observado através dos fragmentos transcritos abaixo:

"Sistematizar a assistência de enfermagem é atuar sob a luz da ciência, estabelecer rotinas para alcançar os resultados estabelecidos. Raciocinar, testar, aplicar os métodos sob os quais a enfermagem atua em seu cotidiano."

(Enfermeiro P) 
"Uma forma de otimizar e organizar a assistência de enfermagem, de forma que as ações sejam padronizadas."

(Enfermeiro C)

As narrativas demonstram, em sua totalidade, o domínio e a confirmação acerca da abordagem sobre a SAE durante o processo de formação profissional, fato que se valida uma vez que o PE foi apresentado no Brasil pela Enfermeira Wanda de Aguiar Horta, no ano de 1979, há 35 anos, ou seja, tempo superior ao de formação declarado pelos profisssionais atuantes na instituição (entre 02 e 29 anos).

Visto isso, foi pedido aos participantes que apontassem as fases do PE, o que comprovou que grande parte da amostra $(71 \%)$ soube citar todas as fases de maneira correta, sendo um fator positivo como referência ao cuidado do paciente, como ilustrado abaixo (figura 2):

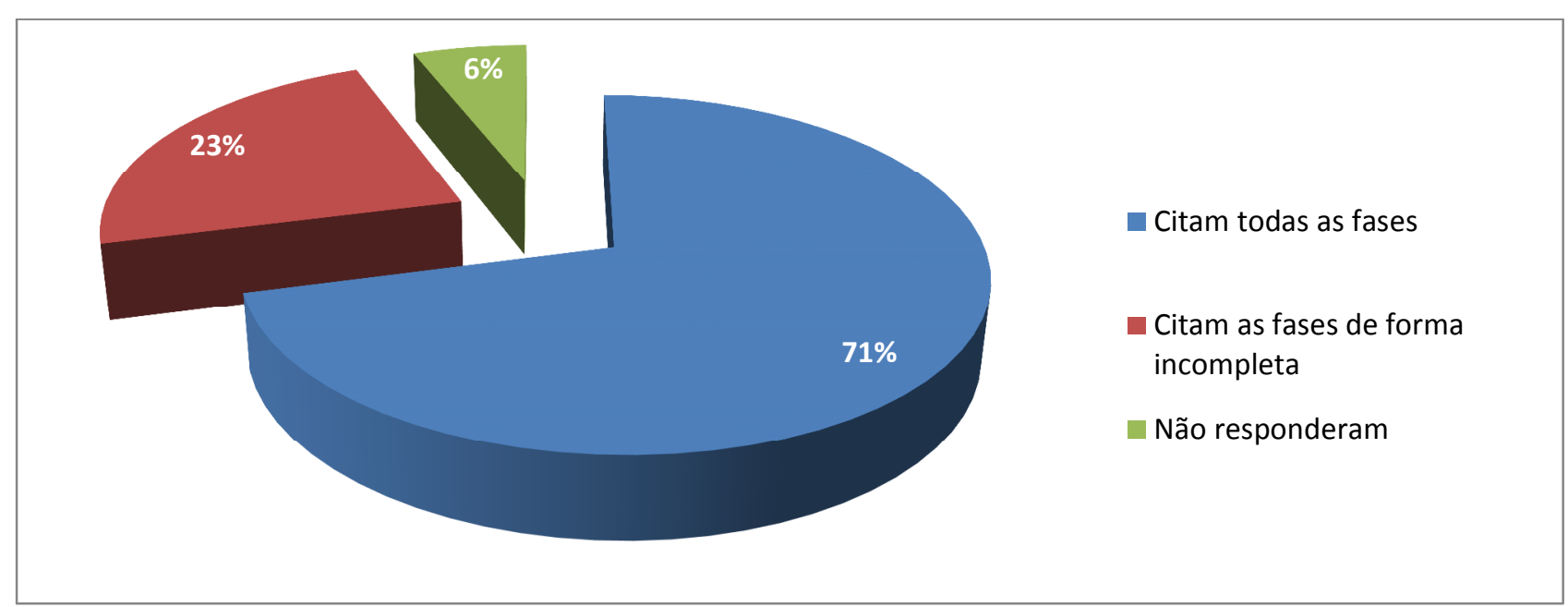

\section{Figura 2 - Conhecimento dos enfermeiros a respeito das fases do Processo de Enfermagem - Campos dos Goytacazes, RJ - 2015 \\ Fonte: Autoria Própria}

Dentre as cinco etapas do PE encontra-se a elaboração dos diagnósticos de enfermagem, que irão nortear as prescrições de enfermagem e posteriormente a conduta do profissional. Segundo NANDA (2015), os diagnósticos de enfermagem são julgamentos clínicos sobre as respostas do indivíduo, da família ou da comunidade aos problemas de saúde/processo de vidas vigentes ou potenciais. Os diagnósticos de enfermagem proporcionam a base para a escolha de intervenção de enfermagem, que visam à obtenção de resultados pelos quais o enfermeiro é responsável. Dada a sua relevância, foi solicitado aos respondentes que elaborassem pelo menos três diagnósticos completos de enfermagem. Através dos resultados obtidos, concluímos que apenas 02 (dois) enfermeiros atingiram o que foi proposto de forma correta, expondo falta de domínio por parte da grande maioria, pois apesar de alegarem que houve abordagem sobre a SAE durante a graduação, uma das etapas fundamentais do PE que abrange a formulação de diagnósticos de enfermagem se mostra deficiente.

Outro aspecto que se destacou durante a entrevista foi ao questionar o conhecimento dos enfermeiros acerca do conteúdo da Resolução COFEN-358/2009 onde a SAE é regulamentada. Apenas 12\% dos entrevistados não responderam, e grande parte, equivalente a $76 \%$ da amostra, demonstrou total desconhecimento sobre seu real conteúdo, podendo ser observado nas reproduções a seguir: 
$\underline{\text { (Enfermeiro } \mathrm{H})}$

"Sei da sua existência, porém não me lembro da resolução".

(Enfermeiro K)

Em contrapartida, uma minoria correspondente a $12 \%$ do total da amostra demonstrou domínio a respeito do conteúdo disposto na resolução supracitada, como podemos confirmar através das declarações a seguir:

"É a resolução que regulamenta à consulta de enfermagem, assim como a sistematização da assistência e que diz que é privativo do enfermeiro à realização da mesma e que tem por objetivo uniformizar e sistematizar os cuidados de enfermagem, devendo acontecer em todas as instituições de saúde públicas e provadas".

(Enfermeiro J)

"Cabe ao enfermeiro, seja em instituição privada ou pública a implantação e elaboração do processo, a descrição das etapas".

(Enfermeiro P)

Contudo, o desconhecimento por parte profissionais a respeito da obrigatoriedade da implantação da SAE em serviços públicos e/ou privados de saúde configura-se como mais uma problemática que contribui para o atraso de uma adesão efetiva do método ao processo de trabalho do enfermeiro.

Parafraseando Dos Santos (2014), o enfermeiro precisa ser autêntico e conquistar o seu espaço com mérito, através do uso de seu conhecimento científico específico, e poderá experimentar na SAE a autonomia necessária para desenvolver um trabalho consciente e gratificante atraindo resultados positivos no cuidado dispensado.

\subsection{Categoria 2 - O Enfermeiro frente à Sistematização da Assistência de Enfermagem X Processo de Trabalho da Enfermagem}

A enfermagem é responsável por mediar um processo de trabalho que irá promover a assistência e direcionar o cuidado, proporcionando não somente a segurança ao paciente, como também a dos profissionais envolvidos. A SAE exerce o papel do instrumento de trabalho do enfermeiro, que tem como foco principal a identificação das necessidades do paciente, apontando um plano direcionado ao seu atendimento e cuidado, conduzindo a equipe de Enfermagem nas ações a serem realizadas, é um processo dinâmico onde carece de conhecimento técnico-científico na prática.

A competência do profissional enfermeiro é caracterizada pela abrangência de sua atuação e compreensão de suas responsabilidades e deveres apresentando autonomia da liderança e a vulnerabilidade da própria dinâmica do trabalho em equipe, com as peculiaridades individuais. Para que o enfermeiro realize o processo de enfermagem, é necessário assumir o compromisso com sua aplicação na prática e a responsabilidade em executar suas etapas, orientar a equipe, aprimorar e atualizar seus conhecimentos. (MENEZES, PRIEL \& PEREIRA, 2010) 
Nesse sentido, foi questionado aos entrevistados acerca da importância da SAE no processo de trabalho e os resultados apontaram que 12 (doze) enfermeiros (70\%) a consideravam como sendo de extrema importância, justificando-se com as seguintes transcrições:

"Trabalhamos com pacientes muito graves que necessitam de total atenção da equipe, de modo que existe um aparato tecnológico grande, havendo assim a necessidade de se usar a mesma ferramenta para assistir a todos".

$\underline{\text { (Enfermeiro C) }}$

"Devido às necessidades individuais, minimizar problemas, nortear o atendimento, reduzir riscos de erros e otimizar o atendimento".

(Enfermeiro I)

Ainda sobre a importância da SAE, 04 (23,5\%) profissionais classificaram-na como muito importante e apenas 01 (6\%) como sendo de média importância, utilizando a justificativa a seguir:

“Apenas contribui para a organização da assistência".

(Enfermeiro Q)

Ao perguntarmos se gostariam que a SAE fosse executada no setor contemplando todas as suas etapas, parte dominante da amostra, equivalente a $88 \%$, mostrou-se favorável e, somente 02 enfermeiros (12\%) discordaram, um deles não se justificou, e outro utilizou o seguinte argumento:

"Não é viável".

(Enfermeiro Q)

A possibilidade de inviabilidade relatada na fala do sujeito foi também apontada no estudo de Colaço et.al. (2015), onde é mencionado que a inviabilidade da SAE na UTI se configura pela dinâmica de trabalho dentro de um setor que exige maior preocupação com a execução da assistência direta ao paciente grave, de forma intensa e constante, atribuindo erroneamente, em função disso, papel acessório aos registros ou avaliações detalhadas e embasadas em raciocínio crítico.

\subsubsection{O Processo de Enfermagem e sua Fragmentação na Prática Intensivista}

Durante a caracterização dos sujeitos constatou-se que o quadro de enfermeiros é formado por diaristas e plantonistas, ocasionando uma divisão de tarefas e horários. O enfermeiro diarista, que trabalha 06 horas diárias de segunda a sexta, é responsável por 03(três) das 05(cinco) etapas do PE, sendo elas: diagnóstico, prescrição e avaliação de enfermagem. Ao enfermeiro plantonista, que trabalha 24 horas semanais, cabe a execução da evolução diária do enfermeiro e as demais fases do PE, admissão/histórico e implementação dos cuidados prescritos, segundo informações colhidas.

Ao questionarmos a amostra a respeito do cumprimento do PE no setor e sobre as etapas utilizadas durante sua rotina diária, as respostas mostraram-se divergentes, apesar do setor afirmar que está em fase de operacionalização da SAE, como observado nas declarações a seguir:

"Aqui não diretamente. Existe um impresso de admissão, onde é usado o histórico de enfermagem".

(Enfermeiro C) 
"Não sistematizado".

(Enfermeiro E)

"Como plantonista não utilizo de forma integral, no setor é feito pelos diaristas".

(Enfermeiro H)

"Sim, todas as etapas".

(Enfermeiro O)

Tal situação detectada nos relatos supracitados, onde a equipe reconhece a dimensão da SAE mais não a aplica de forma integral em seu processo de trabalho, pôde ser notada em estudo semelhante realizado por Pimpão et. al (2010), onde eles evidenciam que embora a equipe de enfermagem pesquisada confirme a relevância da elaboração dos registros e da implementação da SAE, como responsabilidades legais do enfermeiro, principalmente para a continuidade e a qualidade da assistência de enfermagem, essa atividade não é cumprida.

Diante desse contexto, o PE mostra-se fracionado, o que desconfigura o método de planejamento de ações como modelo assistencial, uma vez que o mesmo deve ser efetivado de forma integral por cada profissional, cabendo ao enfermeiro, o compromisso de pensar no planejamento de sua assistência em todas as fases visando o cuidado holístico.

\subsubsection{Desafios e Dificuldades na Operacionalização da SAE}

Quando perguntado o motivo da não realização da SAE e as dificuldades enfrentadas na prática diária, os discursos foram diversos, entre eles destacamos:

“Aceitação da equipe e tempo".

(Enfermeiro B)

“Capacitação, demanda de trabalho, tempo hábil”.

(Enfermeiro $\mathrm{H})$

De acordo com Caixeiro, Dargam \& Thompson (2008), muitas vezes, a falta de tempo dos enfermeiros para efetuar os registros de enfermagem é consequência da ausência de prioridade desta tarefa no seu fazer, ou seja, eles realizam certas atividades que, possivelmente, poderiam ser delegadas a outros profissionais, em detrimento daquela que é fundamental ao seu trabalho.

"Conhecimento dos enfermeiros referentes a SAE e dificuldade de aceitação dos técnicos/auxiliares em executar as prescrições de enfermagem".

$\underline{(\text { Enfermeiro J) }}$

A equipe de técnicos /auxiliares em enfermagem é a grande encarregada pela aplicação da prescrição de enfermagem, precisamente por colaborar com a avaliação do paciente no decorrer do cuidado dispensado, e é de extrema importância que o enfermeiro oriente-os acerca da dimensão dos benefícios que a execução correta das prescrições pode oferecer, afim de minimizar a resistência em praticá-las. 
"A dificuldade para implantação da SAE é o(a) enfermeiro(a); talvez por déficit de conhecimento ou até mesmo falta de vontade".

(Enfermeiro P)

Segundo Menezes, Priel \& Pereira (2010) o enfermeiro muitas vezes se encontra diante de uma prática voltada para a burocracia em sua rotina diária, incorporando atividades de forma mecanizada, com perda de estímulo e motivação, o que o torna vulnerável.

Outra justificativa que pode se correlacionar com as dificuldades encontradas para a efetivação da SAE foi que ao questionarmos se foi oferecido algum treinamento ou curso de aperfeiçoamento no setor de terapia intensiva, mais da metade da amostra entrevistada, equivalente a $60 \%$, relatou que não houve treinamento e/ou aperfeiçoamento algum, apesar de $40 \%$ terem afirmado que houve capacitação.

As UTI são ambientes críticos que abrangem pacientes graves, no qual a enfermagem dispensa cuidados durante 24 horas, todos os dias da semana, estabelecendo ligação direta com o paciente. Assim sendo, esses profissionais necessitam estar atualizados continuamente posto que executam procedimentos invasivos com elevado grau de responsabilidade.

Brito, Silva \& Montenegro (2012) afirmam que a educação permanente no serviço da unidade de terapia intensiva contribui para a atualização, aperfeiçoamento e reciclagem dos conhecimentos, promovendo a adaptação do indivíduo para enfrentar situações vivenciadas no cotidiano.

\subsubsection{Benefícios Proporcionados pela Padronização Assistencial}

Pensando na contribuição da SAE para a atuação do enfermeiro, foi solicitado aos respondentes que citassem benefícios proporcionados pela sistematização, com isso os profissionais que citaram mais de um também foram incluídos no gráfico gerado abaixo (figura 2), onde foi possível observar que os profissionais reconhecem as melhorias possibilitadas pela $\mathrm{SAE}$, como também o quão significativo é o progresso da assistência de enfermagem especializada. O gráfico a seguir demonstra os benefícios decorrentes da SAE e a frequência com que foram citados.

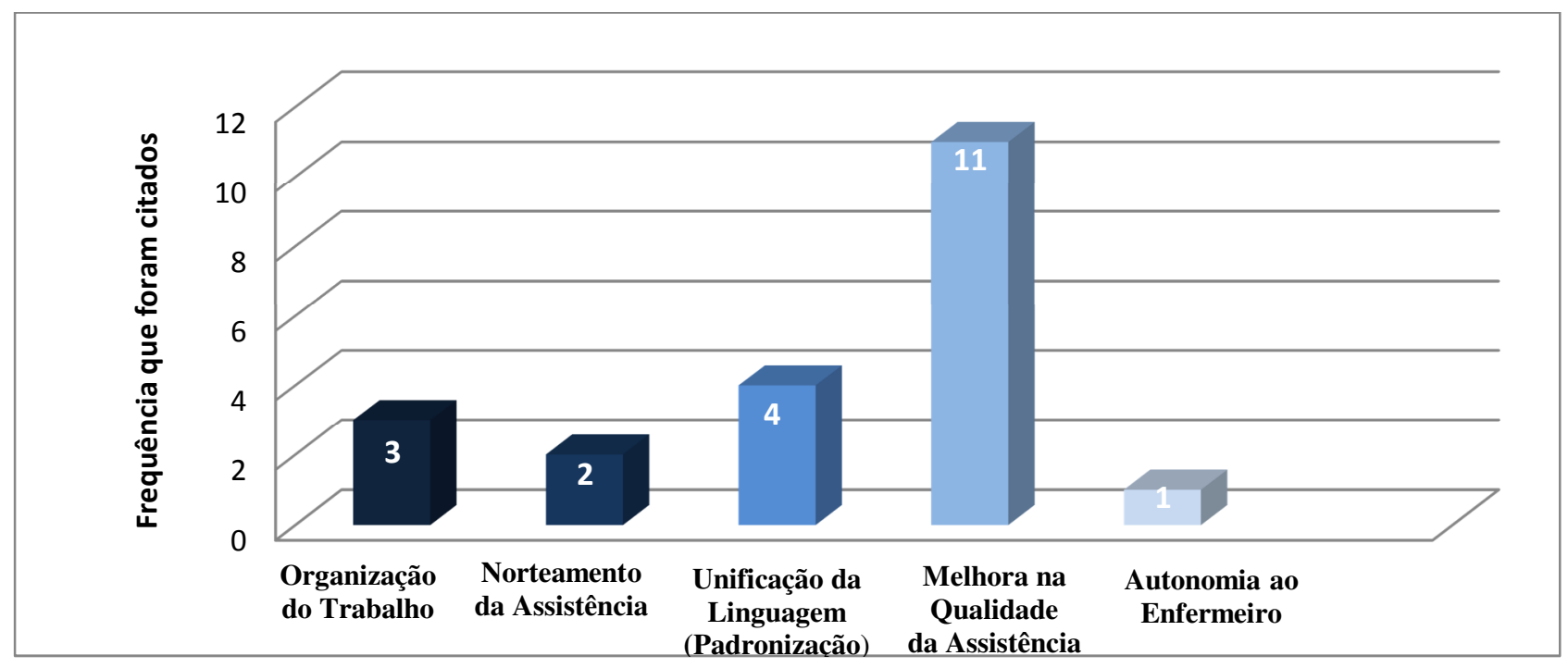

Figura 3 - Benefícios assistenciais proporcionados pela SAE de acordo com os Enfermeiros da UTI. Fonte: Autoria Própria 
Cogo et. al (2012) afirmam que a SAE é uma necessidade que deve se estender do desenvolvimento profissional para o cotidiano do serviço da enfermagem por meio da sua operacionalização, que irá permear a organização, o planejamento de ações sistematizadas de gerenciamento e o cuidado do cliente que está no ambiente hospitalar. A mesma atende às necessidades do paciente em sua complexidade e constrói a autonomia profissional.

Paiano et. al (2014) expõem que a padronização das ações de enfermagem almeja qualificar o cuidado dispensado, oferecer atendimento planejado e individualizado, preservar a organização do setor, otimizar o tempo identificando as prioridades para a tomada de decisões, favorecer a valorização do profissional enfermeiro e consequentemente a valorização da equipe

A SAE é um processo multifacetado na sua implementação, submetido a fatores como: o comprometimento e a motivação da equipe de enfermagem, sobressaindo-se sua importância para o planejamento do cuidado, coordenação do serviço e destaque do papel do enfermeiro. Portanto, é reconhecido que a utilização do PE se mostra como uma tentativa expressiva de padronizar a linguagem entre a equipe, pois, provavelmente, irá oportunizar a excelência no cuidado diário (LAVICH, 2014; INÁCIO et. al, 2014).

\subsection{Categoria 3 - Instrumentos para a Implementação da SAE na UTI: Um Produto de Pesquisa}

Considerando um dos objetivos deste estudo, no que se refere à elaboração de um instrumento de evolução pautado nas fases do $\mathrm{PE}$ e adequado à realidade do setor, os resultados obtidos através das entrevistas e de um check-list da evolução diária do enfermeiro mostraram as maiores dificuldades e desafios para a efetivação da SAE de maneira integral na UTI. Como fragilidades, nos deparamos com a concisão dos formulários preexistentes com limitação de campo para registro, critérios de avaliação escassos e incompletos, relatos de recursos humanos insuficientes, carência de tempo hábil, entre outros. Foi observada que a formulação de instrumentos que sustentam o registro diário do processo de trabalho é deficiente e insatisfatória, pois não abordam todas as necessidades apresentadas por um paciente em estado crítico.

As evidências encontradas possibilitaram a identificação do PE como uma atividade carente de investimentos, postergando sua efetivação de fato. A prática profissional diária é fragmentada e não estimula o reconhecimento sobre a relevância do cuidado fortemente apoiado em evidências.

A ideia de criação e produção de tal instrumento visa subsidiar a assistência diária do enfermeiro ao considerar as principais complexidades e peculiaridades do setor, o adequando assim a sua vivência diária. Partimos de um julgamento clínico de acordo com o que é fundamentado na prática, além de premissas de alguns autores, a fim de gerar um plano assistencial pautado nas fases inter-relacionadas do PE e consequentemente atender as variedades do processo saúde-doença encontradas e suas necessidades de cuidados. A resolução COFEN - 358/2009 dispõe que o PE deve ser constituído basicamente de 05 (cinco) fases, sendo elas: Coleta de dados de Enfermagem (ou Histórico de Enfermagem), Diagnóstico de Enfermagem, Planejamento de Enfermagem, Implementação e Avaliação de Enfermagem, incumbindo ao enfermeiro a liderança durante toda a execução, de modo a alcançar os resultados de enfermagem esperados.

Visto isso, o instrumento elaborado propõe uma possível adoção pelo setor, com o objetivo de auxiliar a assistência dispensada de forma global contemplando todas as premissas indispensáveis em concordância com o Conselho de Enfermagem, para que o PE ocorra e consequentemente a SAE aconteça.

Colaço et. al (2015), destacam a importância da qualidade dos registros a respeito do que o enfermeiro avalia nos pacientes em terapia intensiva, o que tem sido uma característica constantemente apontada e reforçada na formação continuada dos profissionais e, também, na formação de estudantes de enfermagem. Registros objetivos e criteriosos podem inclusive aprimorar a comunicação entre os membros da equipe de saúde. Ressalta-se ainda que registros de excelência podem se estabelecer em garantia do fortalecimento da enfermagem como ciência, além de ser um requisito pertinente do ponto de vista jurídico.

Persp. online: biol. \& saúde, Campos dos Goytacazes, 19 (5), 35-52, 2015

seer.perspectivasonline.com.br 


\section{CONCLUSÕES}

O propósito de implantar a SAE em instituições hospitalares é o de traçar o cuidado a partir da aplicação de um método sistemático, propiciando ao enfermeiro a redefinição da sua área de atuação e aumento no desempenho durante sua assistência. Os autores citados durante o estudo demonstram que a temática da SAE é atual, apesar de ser discutida no Brasil, desde a década de 70, a partir da Enfermeira Wanda de Aguiar Horta.

Com relação à importância da SAE na UTI, evidencia-se a necessidade que o enfermeiro possui de buscar o conhecimento e atualização acercada operacionalização da SAE a fim de que a assistência seja dispensada adequadamente com o objetivo de apoiar com cientificidade e autonomia sua profissão, dado que o PE é um instrumento facilitador e que conduz a uma assistência de qualidade.

No que tange as entrevistas apreendidas, as respostas nos levaram a desvendar os cenários do cotidiano dos enfermeiros onde pudemos desvelar sua atuação na UTI frente à SAE, buscando compreender de que forma este profissional articula e efetiva o PE com sua assistência diária ao paciente em estado grave. Os entrevistados reconhecem a importância da sistematização e citam diversos benefícios como: melhora na qualidade da assistência, padronização da linguagem, organização do trabalho, norteamento da assistência e autonomia ao enfermeiro. Porém, evidencia-se uma fragmentação no seu processo de trabalho, onde este profissional, por tantos motivos, não a concretiza.

Por conseguinte, mediante aos resultados apresentados, pôde-se compreender que existem tanto desafios quanto facilidades atravessando a vivência do enfermeiro frente à operacionalização da SAE, entretanto os desafios se sobrepõem no que se refere às dificuldades de implantação. Alguns entraves encontrados compreendem: implementar a SAE de maneira completa, desconhecimento acerca de sua obrigatoriedade, impressos e protocolos parciais, pouco incentivo a programas de educação permanente, registros de enfermagem incompletos, escassez de tempo hábil, dificuldade de aceitação da equipe de enfermagem e desmotivação.

Nesse contexto, como alternativa para viabilizar a realização dos registros de enfermagem e, consequentemente favorecer o processo de operacionalização da SAE, foi elaborado um instrumento de evolução do enfermeiro pautado nas fases descritas como obrigatórias pelo órgão regulamentador da classe e aplicado à realidade da instituição, pois de nada adianta utilizar a SAE sem que antes haja uma avaliação da realidade que se pretende aplicá-la. Espera-se com este instrumento,uma possível adoção pelo hospital onde a pesquisa foi realizada, no intuito de proporcionar economia de tempo e praticidade, visando uma assistência de qualidade, além de respaldar legalmente as ações de enfermagem, cabendo ao enfermeiro,a iniciativa em aplicar e visualizar o resultado de suas ações devido à importância e as vantagens de sua utilização.

Pretende-se com esta pesquisa, que os profissionais de enfermagem compreendam a relevância da utilização da SAE por meio do PE na UTI, em prol de oferecer umaassistência com qualidade ao paciente crítico, sendo integral e humanizado, portando total autonomia enquanto enfermeiro e fortificando cientificamente sua profissão.

\section{REFERÊNCIAS}

ALVES, A. R.; LOPES, C. H. A. F.; JORGE, M. S. B. Significado do processo de enfermagem para enfermeiros de uma unidade de terapia intensiva: uma abordagem interacionista. Revista da Escola de Enfermagem da USP, v. 42, n. 4, p. 649-55, 2008. 
AMANTE, L. N.; ROSSETTO, A. P.; SCHNEIDER, D. G. Sistematização da assistência de enfermagem em unidade de terapia intensiva sustentada pela teoria de Wanda Horta.Revista da Escola de Enfermagem da USP, v. 43, n. 1, p. 54-64, 2009.

ANDRADE, J. S. de; VIEIRA, M. J. Prática assistencial de enfermagem: problemas, perspectivas e necessidade de sistematização. Revista Brasileira de Enfermagem, v. 58, n. 3, p. 261-5, 2005.

BARDIN, Laurence. Análise de conteúdo. São Paulo: Edições 70, 2011, 229 p.

BARROS, A. L. B. L.; LOPES, J. L. A legislação e a sistematização da assistência de enfermagem. Enfermagem em foco, v. 1, n. 2, 2011.

BRASIL, Ministério da Saúde. ANVISA.Resolução - RDC n 26, de 14 de maio de 2012. Disponível em: $<$ http://www.amib.org.br/fileadmin/user_upload/amib/rdc26_01.pdf> $>$. Acesso em 14 nov. 2015.

BRASIL, Ministério da Saúde. ANVISA.Resolução-RDC n 7, de 24 de fevereiro de 2010. Disponível em: $<$ http://www.amib.org.br/pdf/RDC-07-2010.pdf $>$. Acesso em: 25 out. 2014.

BRASIL, Ministério da Saúde. Política Nacional de Educação Permanente em Saúde. Brasíla: 2009. Disponível em: $<$ http://portal.saude.gov.br/portal/saude/profissional/visualizar texto. cfm? idtxt=27031 $>$. Acesso em: 25 out. 2014.

BRITO, C.G. N. S.; SILVA, N. C.; MONTENEGRO, L. Metodologia de Paulo Freire no desenvolvimento da educação permanente do enfermeiro intensivista. Enfermagem Revista. v. 16, n. 3, p. 317-323, 2012.

CAIXEIRO, S. M. O.; DARGAM, B.; THOMPSON, G. N.Comunicação escrita: importância para os profissionais de enfermagem nas salas de pré-parto. Revista de Enfermagem UERJ, v. 16, n. 2, p. 218-223, 2008.

CENDÓN, B. V.; MEIRELES, M. R. G. Categorização e Classificação de documentos a partir de suas citações: uma proposta baseada em Redes Neurais Artificiais. Pesquisa Brasileira em Ciência da Informação e Biblioteconomia, v. 7, n. 1, 2010.

CIANCIARULO, T. I. et al. Sistema de assistência de enfermagem: evolução e tendências. 4.ed. São Paulo: Icone, 2008.

COFEN.Resolução 358/2009. Dispõe sobre a Sistematização da Assistência de Enfermagem e a implementação do Processo de Enfermagem em ambientes, públicos ou privados, em que ocorre o cuidado profissional de Enfermagem, e dá outras providências. Rio de Janeiro, 2009. Disponível em: $<\underline{\text { http://www.cofen.gov.br/resoluo-cofen-3582009 4384.html> }}$. Acesso em: 15 nov. 2015.

COFEN.Resolução-272/2002. Dispõe sobre a sistematização da assistência de enfermagem (SAE) nas instituições de saúde brasileiras. Rio de Janeiro, 2002. Disponível em: <http://www.corendf.gov.br/portal/index.php/resolucoes/98-resolucao-cofen-2722002>. Acesso em: 25 out. 2014.

COGO, E. et al. Sistematização da assistência de enfermagem no cenário hospitalar: percepção dos enfermeiros. Cogitare Enfermagem, v. 17, n. 3, 2012.

COLAÇO, A. et al. Registro da avaliação de enfermagem em terapia intensiva: Discurso do Sujeito Coletivo. Revista de Enfermagem da UFSM, v. 5, n. 2, p. 257-266, 2015.

DANIEL, L. F. A enfermagem planejada. São Paulo: EPU/DUSP, 1979. 
DE OLIVEIRA, L. M.; EVANGELISTA, R. A. Sistematização da assistência de enfermagem (SAE): excelência no cuidado. Patos de Minas. Revista do Núcleo Interdisciplinar de Pesquisa e Extensão do UNIPAM, v.1, n.7, p. 83-88, 2010.

DOS SANTOS, W. N. et al. Sistematização da Assistência de Enfermagem: o contexto histórico, o processo e obstáculos da implantação. JMPHC.Journal of Management and Primary Health Care, v. 5, n. 2, p. 153-158, 2014.

FREIRE P. Pedagogia do Oprimido. 50 ed. Rio de Janeiro: Paz e terra, 2011.

GARCIA, T. R.; NÓBREGA, M. M. L. Processo de enfermagem: da teoria à prática assistencial e de pesquisa. Escola Anna Nery Revista de Enfermagem, v. 13, n. 1, p. 188-193, 2009.

GIL, A. C. Como elaborar projetos de pesquisa. 4. ed. São Paulo: Atlas, 2008.

HORTA, W. A. Processo de enfermagem. São Paulo: EPU, 1979.

INÁCIO, H. P. et al. A Elaboração de Diagnósticos de Enfermagem e Propostas de Intervenção a um Cliente Submetido à Toracocentese. Revista Rede de Cuidados em Saúde, v. 8, n. 2, 2014.

LAKATOS, E.M.,MARCONI, M. de A. Metodologia científica. 6a ed. São Paulo: Atlas, 2011.

LAVICH, C. R. P. Atuação dos Enfermeiros do Núcleo de Educação Permanente em Enfermagem em um Hospital de Ensino. 2014. Dissertação (Mestrado) - Universidade Federal de Santa Maria, Santa Maria, 2014. Disponível em: $<$ http://coral.ufsm.br/ppgenf/Dissertacao_Claudia\%20Rosane\%20Perico\%20Lavich.pdf $>$ Acesso em: 16 nov. 2015

LUIZ, F. F. et al. A sistematização da assistência de enfermagem na perspectiva da equipe de um hospital de ensino. Revista eletrônica de enfermagem, v. 12, n. 4z, p. 655-9, 2010.

MALUCELLI, A. et al. Sistema de informação para apoio à Sistematização da Assistência de Enfermagem. Revista brasileira de enfermagem. v. 63, n. 4, p. 629-636, 2010.

MASSAROLI, A.; MASSAROLI, R.; MARTINI, J. G. Sistematização da Assistência de Enfermagem nas Unidades de Terapia Intensiva. 17 SENPE-SEMINÁRIO NACIONAL DE PESQUISA EM ENFERMAGEM. 2013, Natal/RN. Seminário. Disponível em:

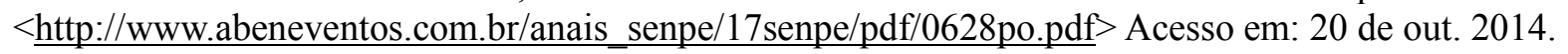

MENEZES, S. R. T.; PRIEL, M. R.; PEREIRA, L. L. Autonomia e vulnerabilidade do enfermeiro na prática da Sistematização da Assistência de Enfermagem. Revista da Escola de Enfermagem da USP, v. 45, n. 4, p. 953-958, 2011.

NANDA.Diagnóstico de Enfermagem NANDA - Definições e Classificações, 2015-2017. 10 ed. Trad. Regina Machado Garcez, Porto Alegre: Artmed, 2015.

NEVES, S. R.; SHIMIZU, H. E. Análise da implementação da Sistematização da Assistência de Enfermagem em uma unidade de reabilitação. Revista Brasileira de Enfermagem, v. 63, n. 2, p. 222-229, 2010.

OLIVEIRA, A. A. P. de. Análise documental do processo de capacitação dos multiplicadores do projeto "Nossas crianças: Janelas de oportunidades" no município de São Paulo à luz da Promoção da Saúde. 2007. 210p. Dissertação (Mestrado) - Escola de Enfermagem, Universidade de São Paulo, São Paulo, 2007. 
PAIANO, L. A. G. et al. Padronização das ações de enfermagem prescritas para pacientes clínicos e cirúrgicos em um hospital universitário. Revista de Enfermagem do Centro-Oeste Mineiro,v.3, n.4, p. 1336-1348, 2015.

PEDREIRA, M.L.G. Práticas de enfermagem baseadas em evidências para promover a segurança do paciente. São Paulo: Acta, 2009.

PIMPÃO, F. D. et al. Percepção da equipe de enfermagem sobre seus registros: buscando a sistematização da assistência de enfermagem. Revista de Enfermagem da UERJ. Rio de Janeiro. p. 10-405, 2010.

POLIT, D. F.; BECK, C. T.; HUNGLER, B. P. Fundamentos de pesquisa em enfermagem: métodos, avaliação e utilização. Trad. de Ana Thorell. 7. ed. Porto Alegre: Artmed, 2011.

REMIZOSKI, J.; ROCHA, M. M.; VALL, J. Dificuldades na implantação da sistematização da assistência de enfermagem-SAE: uma revisão teórica. Cadernos das Escolas de Saúde. n. 03, 2010.

ROCHA, A. M. et al. Análise das necessidades de assistência de enfermagem de pacientes internados em um centro de terapia intensiva para adultos. REME - Revista mineira de Enfermagem.v. 16, n. 3, p. 429-436, 2012.

RUTHES, R.M.; CUNHA, I.C.K.O. Gestão por Competências nas Instituições de Saúde: Uma Aplicação Prática. São Paulo: Ed. Martinari, 2008. 200p.

SANTOS, J. S.; LIMA, L. M.; MELO, I. A. Sistematização da assistência de enfermagem na unidade de terapia intensiva: revisão bibliográfica. Caderno de Graduação-Ciências Biológicas e da Saúde-UNIT v. 2, n. 2, p. 59-68, 2014.

TAKAHASHI, A. A. et al. Dificuldades e facilidades apontadas por enfermeiras de um hospital de ensino na execução do processo de enfermagem. Acta Paulista de Enfermagem. v. 21, n. 1, p. 32-8, 2008.

TANNURE, M. C.; GONÇALVES, A. M. P. Sistematização da Assistência de Enfermagem: Guia Prático. 2 ed. Rio de Janeiro: Guanabara Koogan, 2010. 168p.

TAVEIRA, A. C. F. et al. Premissas de Humanização e Assistência de Enfermagem em Unidade de Terapia Intensiva. In: III SEMINÁRIO DE PESQUISA E TCC DA FUG, 2012-1, Trindade/GO. Seminário.Disponível em: $<$ http://fug.edu.br/2010/pdf/tcc/PREMISSAS\%20DE\%20HUMANIZACAO\%20E\%20ASSISTENCIA $\% 20$ DE\%20ENFERMAGEM.pdf > . Acesso em: 25 de out. 2014.

TRANQUITELLI, A. M.; CIAMPONE, M. H. T. Número de horas de cuidados de enfermagem em Unidade de Terapia Intensiva de Adultos. Revista da Escola de Enfermagem da USP, v. 41, n. 3, p. 371-7, 2007.

TRUPPELL, T. C. et al. Sistematização da Assistência de Enfermagem em Unidade em Unidade de Terapia Intensiva. Revista Brasileira de Enfermagem, v. 62, n. 2, p. 221-227, 2009. 